\section{$\mathbf{M} \mathbf{S}$}

médecine/sciences $1994 ; 10: 250-2$

\title{
LES ANTISENS : SCIENCE ET PATIENCE...
}

\section{Jean-Jacques Toulmé}

\section{ADRESSE}

J.-J. Toulmé : directeur de recherche Inserm; directeur de l'U. 386 de l'Inserm. Laboratoire de biophysique moléculaire, université de Bordeaux Il, 146, rue Léo-Saignat, 33076 'approche antisens repose sur un pari: l'association d'un ARN messager cible et de la séquence complémentaire, dite antisens, va interférer avec la transmission de l'information portée par la cible. Il s'agit donc d'un moyen simple pour engendrer des inhibiteurs de l'expression d'un gène donné, puisqu'il repose sur les règles d'édification de la double hélice d'ADN par formation de paires de bases, connues depuis 1953 [1].

Un examen rapide de la littérature scientifique récente montre que le pari est gagné : les journaux foisonnent d'exemples d'inhibition de l'expression d'un gène par des séquences antisens. Stimulées par l'explosion technologique en biologie moléculaire et en chimie des acides nucléiques, les recherches sont aujourd'hui bien loin des travaux des pionniers de la fin des années 1960. Si les chimistes de l'Institut dirigé par D. Knorre à Novossibirsk [2] et du groupe de P. Ts'o et P. Miller à Baltimore [3] ont, les premiers, envisagé l'emploi d'oligonucléotides antisens, la découverte de mécanismes naturels de régulation de l'expression de gènes procaryotes par des ARN a encouragé les biologistes à engendrer in situ, par transcription de gènes antisens, des ARN complémentaires de l'ARN sens $[4,5]$.

Il existe aujourd'hui plusieurs possibilités pour bloquer artificiellement l'expression d'un gène: ARN ou oli- gonucléotides antisens, triple hélice*, ribozymes. Toutes dérivent conceptuellement de la stratégie antisens: une région d'un ARN ou d'un ADN est ciblée par une séquence oligo- ou polynucléotidique complémentaire, afin de bloquer la réplication ou la transcription de l'ADN, la maturation des pré-ARN, la traduction des ARN messagers ou la transcription inverse des ARN rétroviraux. Plus récemment, une approche sens a été proposée: la cible est cette fois protéique. Les protéines visées assurent une fonction dans l'expression des gènes via l'interaction avec une séquence d'acide nucléique. Les oligonucléotides sens ont la même séquence que le site reconnu par la protéine et agissent donc comme des leurres.

Deux articles, dans ce numéro de médecine/sciences, sont consacrés aux oligonucléotides synthétiques antisens et sens. Les recherches vouées à ces molécules ont considérablement changé au cours de ces dernières années, à la fois en nature et en volume. L'industrie a pris le relais des laboratoires académiques. Nombreuses sont les sociétés de biotechnologie qui se sont créées autour de ces approches. De leur

\footnotetext{
* Cette approche qui repose sur la constitution d'un triple trin local ì partir d'une cible ADN en double brin est parfois appelée "antigène", vocable malheurax, prisque source de comfusion, du moins en français.
} 
côté, les grands groupes pharmaceutiques s'intéressent à ces nouveaux développements. Deux des auteurs des articles sur les antisens, dans ce numéro, illustrent d'ailleurs cet aspect puisque, issus du monde universitaire, ils exercent désormais des responsabilités importantes dans le domaine industriel. C'est dire si les acteurs économiques croient aux perspectives d'application de ces molécules, hors du laboratoire de recherche fondamentale.

Si l'on excepte les travaux des chimistes russes et américains évoqués plus haut, et la contribution remarquable de P. Zamecnik qui, en 1978, a démontré le potentiel des oligonucléotides antisens dans des systèmes complexes [6], les scientifiques n'ont réellement porté attention à cette approche qu'au milieu des années 1980. En moins de dix ans, on est passé du tube à essais, à la culture de cellules puis à l'organisme vivant. Combien, parmi les pionniers des oligonucléotides antisens, auraient prédit que ces molécules feraient l'objet d'essais de phase I chez l'homme en 1993? Les oligonucléotides testés dans ces essais ne sont pas des oligomères naturels. Il s'agit d'oligophosphorothioates, oligomères modifiés sur le squelette pour leur conférer une résistance aux nucléases (voir [7] pour une revue sur les analogues).

Des chercheurs du Centre médical de l'université du Nebraska et de Lynx Therapeutics Inc. ont engagé une étude sur des patients atteints de leucémie myéloïde aiguë. L'oligonucléotide, un 20-mère en série phosphorothioate, est complémentaire d'un segment de l'exon 10 du gène de la $\mathrm{p} 53$. Des études in vitro préalables avaient montré que cet oligonucléotide inhibait la croissance des cellules leucémiques en culture. Un premier essai, datant de 1992, récemment étendu à cinq patients, a permis d'accéder aux premières données pharmacologiques et pharmacocinétiques chez l'homme. Les premiers résultats de l'administration de cette molécule viennent d'être publiés dans le dernier numéro d'Antisense Research and Deve$\mathrm{m} / \mathrm{s} n^{\circ} 3$ vol. 10, mars 94 lopment [8]. Les patients ont reçu, pendant dix jours, $0,05 \mathrm{mg} / \mathrm{kg} / \mathrm{h}$ d'oligonucléotide phosphorothioate anti-p53, en perfusion continue. Aucune toxicité n'a été décelée au niveau clinique selon les critères définis par le National Cancer Institute (toutefois le traitement a été interrompu au sixième jour pour un patient qui avait montré une augmentation du taux d'aspartate aminotransférase et de phosphatase alcaline). Une part importante (30\% à $62 \%)$ de la dose a été retrouvée dans les urines, dont un tiers constitué par de l'oligonucléotide intact. La mise en culture (sur six semaines) d'échantillons de moelle osseuse et de sang périphérique de ces patients a montré une réduction de la croissance cellulaire après traitement.

Dans une autre étude, initiée en France par la société Hybridon Inc., dans le cadre d'une action de l'ANRS, un oligonucléotide phosphorothioate, baptisé GEM91, est en essai de phase I chez vingt-quatre patients asymptomatiques séropositifs pour le VIH. GEM91, long de 25 nucléotides, est complémentaire de la région d'initiation de la traduction du gène gag du VIH. Cette molécule a montré une efficacité remarquable en culture in vitro: dans une étude comparative sur trois isolats différents de VIH-1, elle s'est révélée aussi efficace à la concentration $2 \mu \mathrm{M}$ que l'AZT à $5 \mu \mathrm{M}$ pour bloquer le développement du virus pendant vingt jours (S. Agrawal, Hybridon Inc., communication personnelle). Des études de toxicologie et de pharmacocinétique, chez le rat et le singe, n'ont pas montré d'effets secondaires inquiétants. L'analyse finale de l'essai de phase I devrait être réalisée très prochainement.

D'autres essais sont en cours, ou seront entrepris très prochainement, aux États-Unis. S'il est encore trop tôt pour affirmer que les oligonucléotides antisens constitueront de nouveaux agents thérapeutiques, les résultats actuels n'ont pas fait apparaître d'obstacle majeur à la poursuite des travaux dans cette perspec- tive. Le choix des maladies traitées - leucémie myéloïde aiguë, SIDA - a d'ailleurs surtout été dicté par des aspects d'urgence et de lacunes thérapeutiques. On peut cependant estimer que les oligonucléotides antisens seraient mieux adaptés à des traitements topiques qu'à des traitements systémiques, tels que ceux exigés par la leucémie myéloïde aiguë et le SIDA.

Il ne faudrait pas penser que la situation actuelle soit un aboutissement. Les oligophosphorothioates ne seront certainement pas les seules molécules intéressantes. A quoi ressembleront les prochains candidats antisens aux essais cliniques? Je ne suis pas un "antisensologiste" insensé et ne donnerai pas de réponse à cette question ; pourtant, «spécifique " et "rationnel » sont les qualificatifs les plus fréquemment associés à la description du mode d'action et de la conception des oligonucléotides antisens. Cela découle des règles d'appariement des bases nucléiques. Une cible étant choisie, on en déduit la séquence complémentaire; c'est rationnel. Et, bien sûr, la séquence antisens ne s'associera qu'à la séquence cible; c'est spécifique. Le problème de la sélectivité des effets des oligonucléotides antisens est discuté dans l'article de C. Hélène et E. Saison. Les choses ne se limitent pas au couple cibleantisens. Les effets des RNases $\mathrm{H}$ sur l'inhibition de la traduction ou de la transcription inverse par des oligonucléotides antisens sont bien connus [9, 10], et l'efficacité des oligophosphorothioates contre le VIH provient, en partie, d'effets nonantisens (non spécifiques de séquence). Il a été très récemment montré qu'un oligonucléotide phosphorothioate provoquait une stimulation immune [11] alors que la séquence complémentaire était sans effet...

La conception rationnelle suppose que l'on ait paramétré l'ensemble du système*. Le rationnel s'arrête à

\footnotetext{
* Ie fait que l'on ne puisse actuellement prédire quelle est la meilleure région cible sur un $A R N m$ $n$ 'incite-t-il pas i un peu de modestie?
} 
l'enchaînement des bases*. L'empirisme commence à la conception du squelette qui porte ces bases (puisqu'il semble acquis que l'on ne pourra utiliser des oligonucléotides - ADN ou ARN - conventionnels), et continue par l'association à différents ligands, destinés au ciblage cellulaire ou à augmenter l'internalisation des oligonucléotides, par exemple. Cette remarque vaut aussi pour les oligonucléotides sens décrits par M.Blumenfeld et M. Vasseur.

Ces derniers commentaires n'ont certes pas pour but de refréner l'enthousiasme. Les oligonucléotides antisens ont déjà montré leur formidable potentiel in vivo comme outil de génétique moléculaire, notamment dans le domaine des neurosciences [12]. Il faudra encore beaucoup d'efforts - de science - pour comprendre leur mode d'action, leur mode de pénétration dans les cellules, leur distribution intracellulaire, pour identifier les protéines qui, telles les RNases $H$, contribuent, positivement ou négativement, aux effets antisens. Patience

\footnotetext{
* Bien que l'on envisage l'utilisation de bases non naturelles, en particulier dans la stratégie triplex pour permettre à un trisième brin de "lire "toute séquence en double brin.
}

\section{RÉFÉRENCES}

1. Watson JD, Crick FHC. Molecular structure of nucleic acids. A structure for deoxyribose nucleic acid. Nature 1953; 171: $737-8$.

2. Knorre DG, Vlassov VV. Complementary addressed (sequence-specific) modification of nucleic acids. Progr Nucleic Acids Res Mol Biol 1985 ; 32 : 291-320.

3. Barrett JC, Miller PS, Ts'o POP. Inhibitory effect of complex formation with oligodeoxynucleotide ethyl phosphotriesters on transfer ribonucleic acid aminoacylation. Biochemistry $1974 ; 13$ : 4897-906.

4. Inouye M. Antisense RNA : its functions and applications in gene regulation. A review. Gene 1988; 72: 25-34.

5. Nellen W', Lichtenstein C. What makes an mRNA anti-sense-itive? Trends Biochem Sci $1993 ; 18: 419-43$.

6. Zamecnik PC, Stephenson ML.. Inhibition of Rous sarcoma virus replication and cell transformation by a specific oligodeoxynucleotide. Proc Nall Acad Sci IISA $1978 ; 75: 280-4$.

7. Goodchild J. Conjugates of oligonucleotides and modified oligonucleotides: a review of their synthesis and properties. Bioconjugate Chem 1990; 1: 165-87.

8. Bayever E, Iversen PI., Bishop MR, Sharp JG, Tewary HK, Arneson MA, Pirruccello S], Ruddon RW, Kessinger A, Zon $\mathrm{G}$, Armitage JO. Systemic administration of a phosphorothioate oligonucleotide with a sequence complementary to p53 for acute myelogenous leukemia and myelodysplastic syndrome: initial results of a phase I trial. Antisense Res Dev 1993; 3 : 383-90.

9. Boiziau C, Thuong NT, Toulmé IJ. Mechanisms of the inhibition of reverse transcription by antisense oligonucleotides. Proc Nall Acad Sci USA 1992; 89: 768-72.

10. L.arrouy B, Blonski C, Boiziau C, Stuer M, Moreau S, Shire D, Toulmé IJ. RNase-H mediated inhibition of translation by antisense oligodeoxynucleotides: a way to improve specificity. Gene 1992; 121 : 189-94.

11. McIntyre KW, Lombard-Gillooly K, Perez JR, Kunsch C, Sarmiento UM, Larigan JD, Landreth KT, Narayanan R. A sense phosphorothioate oligonucleotide directed to the initiation codon of transcription factor NF- $\mathrm{KB}$ p65 causes sequencespecific immune stimulation. Antisense Res Dev 1993 ; 3 : 309-22.

12. Wahlestedt C, Golanov E, Yamamoto $\mathrm{S}$, Yee $\mathrm{F}$, Ericson $\mathrm{H}$, Yoo H, Inturrisi CE, Reis DJ. Antisense oligodeoxynucleotides to NMDA-R1 receptor channel protect cortical neurons from excitotoxicity and reduce focal ischaemic infarctions. Nature 1993; 363: 260-3. 